\title{
ON SOME UNRESOLVED QUESTIONS IN CAPITAL THEORY: AN APPLICATION OF SAMUELSON'S CORRESPONDENCE PRINCIPLE*
}

\author{
EDWIN BURMEISTER \\ NGO VAN LONG
}

I. Introduction and summary, 289.-II. The basic model, notation and assumptions, and analysis of the $n=1$ case, 296.-III. The no-joint production case with $n$ $>1,301$.-IV. The joint production case with $n>1,303$. -V. The social rate of return, 306.-VI. Concluding remarks, 312.

\section{INTRODUCTION AND SUMMARY}

In the mid-1960's two quite separate but fundamental questions in capital theory were first debated in this Journal. On the one hand, the original paper by Levhari (1965) gave rise to the widely cited series of papers published jointly in November, 1966, as "Paradoxes in Capital Theory: A Symposium," which has since spawned dozens of articles and several books. ${ }^{1}$ This debate now constitutes a crucial part of "The Cambridge Controversy." On the other hand, that same November, 1966, issue of this Journal also contained Hahn's seminal paper on the dynamic properties of heterogeneous capital good models, and subsequent research on "the Hahn problem" has been voluminous, continuing to this time. ${ }^{2}$

The issues are different. The fundamental aspect of the Cambridge controversy we are concerned with in this paper centers on comparisons of alternative steady-state equilibrium positions when the profit or interest rate is varied as an exogenous parameter. Thus, for example, if we confine our attention to interest rates above the Golden Rule value (the exogenous rate of labor force growth $g$ ), it is possible that a fall in the rate of interest results in a fall in steady-state per capita consumption (measured in the per capita number of fixed consumption baskets). In this case the economy is said to exhibit "paradoxical consumption behavior," the "paradox," of course, being that this consumption behavior contradicts the neoclassical parable.

* E. Burmeister acknowledges with thanks research support from the John Simon Guggenheim Memorial Foundation, the Australian National University, the Center for Advanced Studies at the University of Virginia, and the National Science Foundation.

1. Harcourt (1972), Blaug (1975), and Brown, Sato, and Zarembka (1976) are examples.

2. See, for example, Hahn (1966), Samuelson (1967), Shell and Stiglitz (1967), Kurz (1968), Burmeister and Graham (1974), and Cass and Shell (1976). 
More precisely, the Solow-Swan one-sector model leads us to expect that any economy in a steady-state equilibrium with a lower interest rate (but still above $g$ ) will also have a higher value of steady-state per capita consumption. Furthermore, in the Solow-Swan model, where there is no problem of aggregating bolts and hammers into a single measure of "capital," a lower steady-state interest rate always implies a larger value in the stock of homogeneous machines (Swan's meccano sets or Joan Robinson's (1966) LEETS).

"The Hahn problem" is not concerned with such comparisons of alternative steady-state equilibria positions, but rather it is about the stability of a model that is identical but for the addition of an extra condition to determine simultaneously the interest rate as one (endogenous) variable in a complete general equilibrium system. Generally, any "savings equals investment" equation or, equivalently, any consumption function will suffice as the additional condition; Hahn's original formulation used the Marx-Kalecki assumption that all profits are saved (reinvested) and all wage income is spent on consumption goods, but this is not essential to his conclusions. Thus, given a complete general equilibrium model, "the Hahn problem" is concerned with whether or not the economy is dynamically stable, i.e., whether or not the vector of per capita capital stocks will converge to some (perhaps unique) steady-state equilibrium or rest point. Hahn's startling conclusion was that in general such convergence is not to be expected; a model with heterogeneous capital goods is featured by a generalized saddlepoint equilibrium, and the economy will converge to a steady state (a rest point of the dynamic system) only if the initial values of the $n$ capital stocks and the initial values of their respective prices lie in a restricted subset of the $2 n$-dimensional price-capital space. Since the initial vector of per capita capital stocks is exogenous, this means that only particular and very special choices of the initial price vector will yield convergence. Moreover, if we discard the possibility of perfect markets for every commodity into the infinite future as unrealistic in any descriptive model, ${ }^{3}$ then there is nothing in the economic system to assure that the initial price vector will be consistent with convergence. One is left with the uncomfortable conclusion that dynamic heterogeneous capital good models exhibit "Hahn instability." (Perhaps uncertainty will provide one resolution of this difficulty, but the work to date is fragmentary; Burmeister and Graham (1974) illustrate one conceivable approach.)

As already noted, the structures of the economic models used to analyze questions pertaining to the Cambridge controversy and the

3. See Samuelson (1967) and Shell-Stiglitz (1967). 
Hahn problem are identical, except that for the former questions the interest rate is taken as an exogenous parameter (which varies to generate alternative steady-state equilibria), while for the latter stability question a savings-investment condition must be supplemented so that the interest rate is determined endogenously. This formal similarity is enough to suggest that there exists some relationship between paradoxical behavior and Hahn instability. More precisely, it is natural to conjecture a form of Samuelson's Correspondence Principle; ${ }^{4}$ that is, to conjecture that steady-state points that exhibit paradoxical behavior are dynamically unstable in some sense, and conversely. To illustrate that this conjecture is an exact application of the Correspondence Principle, at least when the labor force is constant, we quote Samuelson's original statement:

In a previous paper ${ }^{5}$ it was pointed out that there exists an intimate formal dependence between comparative statics and dynamics. To my knowledge this had not previously been explicitly enunciated in the economic literature, and for lack of a better name I shall refer to it as the Correspondence Principle. It is the purpose here to probe more deeply into its analytical character, and also to show its two-way nature: not only can the investigation of the dynamic stability of a system yield fruitful theorems in statical analysis, but also known properties of a (comparative) statical system can be utilized to derive information concerning the dynamic properties of a system. ${ }^{6}$

The notion that some Correspondence Principle provided a logical connection between the Cambridge controversy and the Hahn problem was implicit in private conversations around M.I.T. in the later 1960's, but to our knowledge the only published version of this idea is Solow's statement in his Foreword to Burmeister and Dobell (1970):

Third, there are problems connected with the "paradoxes" discussed by Burmeister and Dobell in Chapter 8. The key difficulty may be one of interpretation. The paradoxes themselves show that some simple conclusions deduced from models with one capital good need not hold from more general models, but it remains to be seen how significant this is. If the paradoxes matter at all, they are likely to matter for this ubiquitous question of convergence to steady states. The simpler question is whether such paradoxes can be observed in an optimizing economy, or whether if an optimal path comes upon such a situation, it will go around it, so to speak, so "paradoxical behavior" will never be observed along an optimal path. In general, even this question remains unanswered, although some particular cases, with especially simple criterion

4. All the conclusions of our analysis here remain valid when the exogenous growth rate of labor $g$ is set equal to zero. In this special case the steady-state comparisons we are making involve comparative statics. (When $g>0$, the alternative comparisons involve comparative dynamics.)

5. Samuelson (1941).

6. Samuelson (1942), p. 1; also see Samuelson (1941). Both the above are reprinted in Samuelson (1966). 
functions, have been studied. Nobody knows what sort of path an unplanned profitmaximizing economy would follow in similar circumstances. These sound like very hard problems, but important ones. ${ }^{7}$

Surprisingly, with the exception of Burmeister and Hammond (1977), not a single paper has been published investigating such a relationship. Thus, although the idea is rather obvious and intuitively appealing, it is not easy to formulate in a precise statement. A careful reading of Samuelson $(1941,1942)$ reveals the basic difficulty: the Correspondence Principle is a statement about a single system of functional equations while, as already noted, the Cambridge controversy and the Hahn problem are concerned with systems that differ with respect to determination of the interest rate. Having noted this difficulty, however, a remedy immediately suggests itself. It is wellknown that the Hahn instability feature of descriptive economic models is closely related to the saddlepoint property of optimal economic models (see, for example, Kurz (1968), Burmeister and Dobell (1970), especially pp. 402-406, and the references cited there). What is possible, therefore, is that we may find a valid Correspondence Principle between paradoxical points and the dynamic stability properties of the rest points to an optimizing problem (rather than to Hahn's descriptive problem). The obvious candidate for the optimizing problem is

$$
\max _{\{k(t)\}} \int_{0}^{\infty} u(c) e^{-\rho t} d t
$$

subject to initial conditions and restrictions imposed by the feasible technology set, where $k$ is a vector of per capita capital stocks, $u(c)$ is instantaneous utility of per capita consumption $c$, and $\rho \geqq 0$ is the exogenous rate of time discount. Two features of the solution to problem (1) are crucial:

(i) Along an optimal trajectory solving (1), $r(t)=\rho+g=r$ is constant for all $t$, where $r(t)$ is the own-rate of return in terms of the numéraire $u(c)$.

(ii) The solution to (1) entails $2 n$ necessary differential equations, namely $n$ differential equations describing the accumulation of the per capita capital stocks and the $n$ differential equations governing the price movements of these capital goods. (These prices are in terms of "utility units" as numéraire and are denoted by the vector $p$.) When the utility function is linear with $u(c)=c$, this set of $2 n$ differential equations is identical to the dynamic equations for the Hahn problem with a fixed value of $r$.

7. Solow's Foreword to Burmeister and Dobell (1970), pp. viii-ix. 
In view of (i) we may consider $\rho$ both as a parameter of problem (1) and as the parameter that is varied to generate the alternative steady-state equilibria discussed in connection with the Cambridge controversy. In this paper we restrict our attention to steady states characterized by $\rho \geqq 0$, which are therefore efficient equilibria in the Phelps-Koopmans sense. Before proceeding, we now must introduce some preliminary notation and definitions. For simplicity we assume that there is a single consumption good. Let $\bar{c}$ and $\bar{k}$ denote steadystate equilibrium values of per capita consumption and the vector of per capita capital stocks, respectively.

DEFINITION 1. A steady-state rest point $(\overline{\mathrm{c}}, \overline{\mathrm{p}}, \overline{\mathrm{k}})$ is not paradoxical if

$$
0 \neq \operatorname{sgn}\left(\frac{\mathrm{d} \overline{\mathrm{c}}}{\mathrm{d} \rho}\right)=-\operatorname{sgn}(\mathrm{r}-\mathrm{g}) \text { for } \rho=\mathrm{r}-\mathrm{g} \neq 0 .
$$

DEFINITION 2. An economy is termed regular if

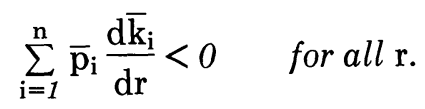

Since

$$
\frac{d \bar{c}}{d \rho}=\rho \sum_{i=1}^{n} \bar{p}_{i} \frac{d \bar{k}_{i}}{d \rho},
$$

an economy is free of paradoxical rest points if and only if it is regular (see Burmeister and Dobell (1970, Ch. 9) and Burmeister and Turnovsky (1972)).

DEFINITION 3. A rest point solution $(\overline{\mathrm{p}}, \overline{\mathrm{k}})$ to problem (1) is called a regular saddlepoint if the associated linear system of the necessary $2 \mathrm{n}$ differential equations has $\mathrm{n}$ characteristic roots with negative real parts and $\mathrm{n}$ characteristic roots with positive real parts. It is called an unstable saddlepoint if more than $\mathrm{n}$ such roots have positive real parts and if none have a zero real part.

Suppose that a rest point solution $(\bar{p}, \bar{k})$ to problem (1) is a regular saddlepoint. Then for given initial capital stocks sufficiently close to the $\bar{k}$ equilibrium, there exists a unique set of $n$ initial prices satisfying the necessary conditions for the optimization problem (1) such that the unique solution path $\{p(t), k(t)\}$ converges asymptotically to $(\bar{p}, \bar{k})$. On the other hand, if $(\bar{p}, \bar{k})$ is an unstable saddlepoint, then given initial capital stocks sufficiently close to the equilibrium $\bar{k}$, the 
optimizing path $\{p(t), k(t)\}$ converges to $(\bar{p}, \bar{k})$ only if the given initial capital stocks happen to be in certain very special ratios.

Equipped with these definitions, we are able to state precisely a candidate Correspondence Principle:

\section{Conjecture CP (Correspondence Principle)}

An efficient steady-state point $(\bar{c}, \bar{p}, \bar{k})$ is paradoxical if and only if it is not a regular saddlepoint with more than $n$ characteristic roots of the associated linear system having positive real parts, i.e., if and only if it is an unstable saddlepoint.

Upon reflection it should be realized that Conjecture CP is too good to be true. If it were valid generally, it would justify nearly all of the conventional neoclassical wisdom based on the Solow-Swan one-sector model. For example, were Conjecture CP valid, it could be argued legitimately that while paradoxical steady-state equilibria are possible, an optimizing economy "almost never" would be observed at (or near) such a rest point because, as we shall argue below, that point would be an unstable saddlepoint in the sense of Definition 3. Likewise, as we shall prove in Section V below, were Conjecture CP valid, transitions between steady-state equilibria based on a naive rule using Solow's concept of the social rate of return would never cause movements away from a saddlepoint-stable rest point solution to problem (1) in the $(\bar{c}, \rho)$ plane.

It is precisely because Conjecture CP is so powerful that it becomes crucial to learn under what conditions it may be valid. In retrospect the answer is not surprising; as we shall prove below as Theorem 1, Conjecture CP is valid when there exists only one kind of capital good, i.e., $\mathrm{n}=1$. While this result will be disappointing to ardent neoclassicists, it is not a trivial proposition because "paradoxical consumption behavior" definitely can occur even when $n=$ 1 , provided that the possibility of joint production is admitted. ${ }^{8}$

For $n>1$ our analysis is divided into two cases: (a) joint production is excluded, and (b) joint production exists. The simplicity of the no-joint production case is due to the fact that for any given $\rho$, a corresponding rest point solution to problem (1), if it exists, must be unique. In case (a) we then are able to prove, under certain assumptions, ${ }^{9}$ that the local stability properties of all rest points are

8. See Burmeister and Turnovsky (1972).

9. Most important, our assumptions must be strong enough to justify a local stability analysis based on the associated linear system. The validity of these linear approximations necessitates that none of the $2 n$ characteristic roots of the associated linear system has a zero real part (thereby excluding both zero and purely imaginary roots). 
identical. If the rest point corresponding to the Ramsey problem with $\rho=0$ is a regular saddlepoint, as will be true for nonpathological problems, it follows that every rest point solution to problem (1) is also a regular saddlepoint. As an immediate consequence of our results, we find that under these conditions unstable points (in the saddlepoint sense) and hence limit cycles are precluded.

In the joint production case there may exist several rest point solutions to problem (1) for the same value of $\rho$ (necessarily positive since the Golden Rule point at $\rho=0$ is unique). Ironically, we are then able to state a valid Correspondence Principle and to prove what in 1942 Samuelson called a "separation" theorem; ${ }^{10}$ our results are quite parallel. ${ }^{11}$

What, then, shall we be able to conclude from our analysis, which follows? First, while the most powerful form of a Correspondence Principle, stated above as Conjecture $\mathrm{CP}$, would justify many results based on the neoclassical parable, it is valid generally only when there is one type of capital good, i.e., $n=1$. Second, when there is no joint production and certain other assumptions are satisfied, every rest point is a regular saddlepoint in the sense of Definition 3. Third, when joint production exists, a Correspondence Principle is valid in that, under certain conditions, a paradoxical point in the sense of Definition 1 is not a regular saddlepoint in the sense of Definition 3. Moreover, a Separation Theorem obtains in that regular saddlepoints are separated from unstable saddlepoints. Finally, if (i) a steady-state point $(\bar{p}, \bar{k})$ is unique for a given $\rho \geqq 0$ (which is always true when joint production is excluded), or (ii) the economy is regular in the sense of Definition 2 (which implies uniqueness of rest points for our model), then it can be shown that, with certain additional assumptions, every steady state is a regular saddlepoint in the sense of Definition 3, thus proving a Correspondence Principle that regularity implies saddlepoint stability. While the conditions under which these statements are true are restrictive, they do seem to include cases of genuine economic interest.

Given that we have demonstrated that optimal dynamics are extremely complex, what can one say with regard to policy? When does a simple rate of return rule lead to qualitatively correct policy advice? Again we show that the Correspondence Principle applies here: For regular economies, a simple rule based on Solow's social rate of return will lead to the correct optimal rest point.

The remainder of this paper is divided into four sections. In

10. "Points of stable equilibrium (in the small) are separated by points of definitely unstable equilibrium; and vice versa." Samvelson (1942), p. 10.

11. See Theorem 5 in Section IV below. 
Section II we introduce the basic model, notation, and assumptions, and we then prove that Conjecture $\mathrm{CP}$ is valid when $n=1$. In Section III we treat the no-joint production case, while the complicated joint production case in which there may exist multiple rest points for the same value of $\rho$ is analyzed briefly in Section IV. Finally, in Section $\mathrm{V}$ we apply our previous results and derive the conditions under which rules based on Solow's social rate of return do or do not provide legitimate criteria for transitions between steady states.

\section{The Basic Model, Notation AND Assumptions, AND ANALYSIS OF THE $n=1$ CASE}

\subsection{The Basic Model, Notation, and Assumptions}

The economy produces $n$ investment goods $Y_{i}(i=1,2, \ldots, n)$ and one consumption good ${ }^{12} C$, using labor $L$, and capital $K_{i}(i=1$, $2, \ldots, n)$, where every factor of production is required, either directly or indirectly, to produce the consumption good and where no output of any commodity is possible without a positive labor input. The labor force grows at a constant rate $g \geqq 0$, and we have

$$
\dot{k}_{i}(t)=y_{i}(t)-\left(g+\delta_{i}\right) k_{i}(t),
$$

where $k_{i}=K_{i} / L, y_{i}=Y_{i} / L$, and $\delta_{i}$ is the exponential depreciation rate for the $i$ th capital good.

The economy's production set is $W \subseteq R_{+}^{2 n+2}$-a set of technologically feasible input-output combinations $(C, Y, K, L) \geqq(0,0,0$, $0)$. $W$ is a cone with vertex 0 , implying constant returns to scale. The per capita production set is

$$
V=\left\{(c, y, k) \subseteq R_{+}^{2 n+1} \mid \exists L,(L c, L y, L k, L) \in W\right\} .
$$

$V$ is clearly convex. Let $D$ be the projection of $V$ on the $(y, k)$ space. For any point $(y, k) \in D$ we define the function,

$$
G(y, k)=\underset{(c, y, k) \in V}{\operatorname{maximum}} c .
$$

In what follows, static efficiency is assumed, so that we shall write

$$
c=G(y, k)
$$

for $(y, k) \in D$. It will be assumed that $G(y, k)$ is a well-behaved concave function, with $G(0,0)=0$ and

12. There is no essential difference if there are many consumption goods. 


$$
\begin{array}{cc}
\partial G / \partial y_{i}<0 & \partial G / \partial k_{i}>0 \\
\partial^{2} G /\left(\partial y_{i}\right)^{2} \leqq 0 & \partial^{2} G /\left(\partial k_{i}\right)^{2} \leqq 0 .
\end{array}
$$

The production set $W$ is said to exhibit no-joint production if it is (or may be regarded as being) generated by $n+1$ neoclassical production functions, as defined by Burmeister and Dobell (1970, pp. 9-10),

$$
\begin{gathered}
C=F^{0}\left(L_{0}, K_{10}, \ldots, K_{n 0}\right) \\
Y_{i}=F^{i}\left(L_{i}, K_{1 i}, \ldots, K_{n i}\right) \quad(i=1,2, \ldots, n) .
\end{gathered}
$$

Some or all of these functions may be identical. Joint production is said to exist if the production set is not generated by individual production functions for every commodity.

Using (2.1) and (2.2), we may write

$$
c=G\left[\ldots, \dot{k}_{i}+\left(g+\delta_{i}\right) k_{i}, \ldots, k_{i}, \ldots\right]
$$

hence

$$
c=F(\dot{k}, k) .
$$

We assume that there exists a unique $k^{*}$ maximizing $F(0, k)$ and that $F\left(0, k^{*}\right)>0$. It can be shown that our assumption that labor is essential implies that all feasible $k_{i}(t)$ paths are bounded. This ensures that each $k_{i}$ is finite in a steady state.

Consider now the problem of choosing $\dot{k}_{i}(t)$ and $c(t)$ so as to solve

$$
\max _{\left\{k_{i}(t)\right\}} \int_{0}^{\infty} e^{-\rho t} u[c(t)] d t
$$

subject to given $\rho \geqq 0$ and

$$
\begin{aligned}
c & =F(\dot{k}, k) \\
k(0) & =k_{0} \\
c(t) & \geqq 0 \\
y_{i}=\dot{k}_{i}+\left(g+\delta_{i}\right) k_{i} & \geqq 0, \quad i=1, \ldots, n,
\end{aligned}
$$

where $u(c)$ is a concave and strictly increasing function. We define

$$
T(\dot{k}, k)=u[F(\dot{k}, k)]=T(x, k), \quad x \equiv \dot{k} .
$$

The Hamiltonian function for the problem (2.4) is

$$
H_{0}(p, k, \dot{k})=T(k, \dot{k})+p \dot{k} .
$$


Let

$$
H(p, k)=\max _{\{\dot{k}\}} H_{0}(p, k, \dot{k})
$$

An optimal trajectory must satisfy $2 n$ differential equations:

$$
\begin{aligned}
& \dot{k}_{i}=\partial H / \partial p_{i} \\
& \dot{p}_{i}=\rho p_{i}-\partial H / \partial k_{i} .
\end{aligned}
$$

Note that for given $(p, k), \dot{k}$ maximizes $H_{0}(p, k, \dot{k})$, implying that

$$
T_{x_{i}}+p_{i} \leqq 0, \quad x_{i} \equiv \dot{k}_{i}
$$

with equality if $\dot{k}_{i}+\left(g+\delta_{i}\right) k_{i}>0$.

Since we are not interested in the problem of nonuniqueness of momentary equilibrium, it will be assumed that the maximization of $H_{0}(p, k, \dot{k})$ for given $(p, k)$ yields a unique vector $\dot{k}$, at least in some neighborhood of any rest point $(\bar{p}, \bar{k})$. Technically, this assumption is satisfied if $T(\dot{k}, k)$ is strictly concave in $\dot{k}$ in some neighborhood $N_{\epsilon}$ of $(\dot{k}, k)=(0, \bar{k})$, or the matrix $T_{x x}$ is negative definite ${ }^{13}$ almost everywhere in $N_{\epsilon}$.

We are interested in the local stability characteristics of rest points, and in particular the relation between instability and "paradoxical" behavior in the sense of Definition 1 . The linear system associated with the system (2.12)-(2.13) is

$$
\left(\begin{array}{c}
\dot{k} \\
\dot{p}
\end{array}\right)=J\left(\begin{array}{l}
k(t)-\bar{k} \\
p(t)-\bar{p}
\end{array}\right),
$$

where $(\bar{p}, \bar{k})$ is a rest point. The entries of matrix $J$ are evaluated at $(\bar{p}, \bar{k})$, and

$$
J=\left(\begin{array}{cc}
H_{p k} & H_{p p} \\
-H_{k k} & \rho I-H_{p k}
\end{array}\right) .
$$

It is interesting, and perhaps suggestive, to note that "paradoxical" steady states are related to a quadratic form involving the matrix

13. To prove that $H_{p p}$ is positive definite if and only if $T_{x x}$ is negative definite, differentiate (2.12) and (2.14), with $d k=0$ :

$$
\begin{gathered}
H_{p p} d p=d \dot{k} \\
-T_{x x} d \dot{k}=d p ;
\end{gathered}
$$

hence

$$
H_{p p}=\left[-T_{x x}\right]^{-1}
$$


$J$. At a steady-state rest point $(\bar{p}, \bar{k})$ we have, from (2.12) and (2.13),

$$
\begin{gathered}
\partial H / \partial p_{i}=0 \\
\rho \bar{p}_{i}-\partial H / \partial k_{i}=0 .
\end{gathered}
$$

Differentiating (2.16) and (2.17) with respect to $\rho$, we obtain

$$
M\left(\begin{array}{c}
p^{\prime} \\
k^{\prime}
\end{array}\right)=\left(\begin{array}{c}
0 \\
-p
\end{array}\right), \frac{d p}{d \rho}=p^{\prime}, \text { etc. },
$$

where it is understood that we have now dropped the bars and denote steady-state values $\bar{p}$ and $\bar{k}$ simply by $p$ and $k$; and where

$$
\begin{aligned}
M & =\left(\begin{array}{cc}
H_{p p} & H_{p k} \\
\rho I-H_{p k} & -H_{k k}
\end{array}\right) \\
& =J\left(\begin{array}{ll}
0 & I \\
I & 0
\end{array}\right) .
\end{aligned}
$$

From (2.19)

$$
\left(p^{\prime T}, k^{\prime T}\right) M\left(\begin{array}{l}
p^{\prime} \\
k^{\prime}
\end{array}\right)=-p^{T} k^{\prime}
$$

where $p^{T}$ denotes the transposed $p$, etc. But

$$
\begin{aligned}
\operatorname{sgn} \frac{d \bar{c}}{d \rho} & =\operatorname{sgn} \frac{d T}{d \rho}=\operatorname{sgn} \Sigma T_{k_{i}}(0, \bar{k}) \frac{d k_{i}}{d \rho} \\
& =\operatorname{sgn}\left(\rho \Sigma p_{i} k_{i}^{\prime}\right) \\
& =\operatorname{sgn}\left(\rho p^{T} k^{\prime}\right) .{ }^{14}
\end{aligned}
$$

\subsection{The $n=1$ Case}

a. No-joint production. In this case, for any given $\rho \geqq 0$, a steady state, if it exists, is unique, and has the regular saddlepoint property. Steady states are nonparadoxical in the sense of Definition 1 (see Theorem 2 below).

b. Joint production case. In this case, for any given $\rho>0$, there may exist more than one steady state. This possibility was considered in Liviatan and Samuelson (1969), where the relationship between instability and $d \bar{k} / d \rho$ was established: for $\rho>0$, steady-state rest points at which $d \bar{k} / d \rho<0$ are regular saddlepoints and those at which $d \bar{k} / d \rho>0$ are unstable in the sense of Definition 3 .

14. See Burmeister and Dobell (1970), pp. 286-87, and Brock and Burmeister (1976) for further discussion. 
We now present a proof of Conjecture CP for the case $n=1$.

THEOREM 1 . For $\mathrm{n}=1$, a rest point $(\overline{\mathrm{c}}, \overline{\mathrm{p}}, \overline{\mathrm{k}})$ is paradoxical if and only if it is not a regular saddlepoint.

Proof. We consider only the case where $\operatorname{det} J \neq 0$ (otherwise local stability analysis based on linearization is not justified). From (2.20) we have $\operatorname{det} J=-\operatorname{det} M$. Now, from (2.18)

$$
\left(\begin{array}{l}
p^{\prime} \\
k^{\prime}
\end{array}\right)=M^{-1}\left(\begin{array}{c}
0 \\
-p
\end{array}\right)
$$

then by direct computation

$$
\begin{aligned}
-p^{T} k^{\prime} & =\left[0,-p^{T}\right] M^{-1}\left(\begin{array}{c}
0 \\
-p
\end{array}\right) \\
& =p^{T} H_{p p} p / \operatorname{det} M .
\end{aligned}
$$

But $H_{p p}=\left[-T_{x x}\right]^{-1}>0$ because $-\infty<T_{x x}<0$ for $c, y>0$. Hence using (2.22), we see that

$$
\frac{d \bar{c}}{d \rho} \gtrless 0 \text { as } \operatorname{det} J \gtrless 0 \text { for } \rho>0 .
$$

For the case $\rho=0$ there exists a unique rest point $\left(p^{*}, k^{*}\right)$. Under our assumption that $\operatorname{det} J \neq 0$, this rest point is a regular saddlepoint with roots $\lambda_{1}=-\lambda_{2} \neq 0$ if $\operatorname{det} J=\lambda_{1} \lambda_{2}<0$. Thus, it suffices to prove that $\operatorname{det} J \leqq 0$; but $\operatorname{det} J=-\operatorname{det} M=H_{p p} H_{k k}-\left(H_{p k}\right)^{2} \leqq 0$, since $H$ is concave in $k$ and convex in $p$; see Samuelson (1972). This completes the proof.

Finally, we establish a property that is true for the $n=1$ case but not true for the more general $(n>1)$ case.

THEOREM 2. For $\mathrm{n}=1$, a paradoxical steady-state point occurs only at values of $\rho$ giving rise to multiple rest points. In other words, $\mathrm{d} \overline{\mathrm{c}} / \mathrm{d} \rho \neq 0$ for $\rho \neq 0$.

Proof. Suppose that there were some $\rho^{0} \neq 0$ at which $d \bar{c} / d \rho=$ 0 . Then $p k^{\prime}\left(\rho^{0}\right)=0$ by (2.22). But $p \neq 0$ by (2.14), and hence $k^{\prime}\left(\rho^{0}\right)$ $=0$. Thus, (2.18) implies that

$$
\begin{gathered}
\left(\rho-H_{p k}\right) p^{\prime}=-p \\
\left(H_{p p}\right) p^{\prime}=0
\end{gathered}
$$

or

$$
H_{p p}=0 .
$$




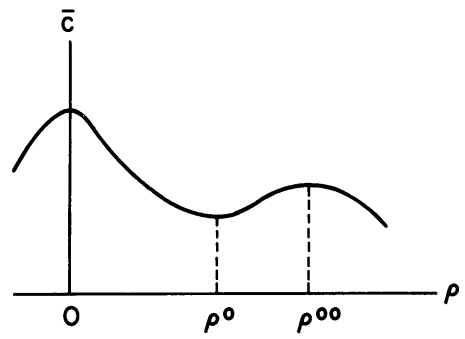

FIGURE I

But $H_{p p}=\left(-T_{x x}\right)^{-1}>0$.

Q.E.D.

Theorem 2 rules out the behavior depicted in Figure I when $n=1$.

\section{The No-Joint Production CASE With $n>1$}

First, we shall show that for any given $\rho \geqq 0$, a steady-state rest point, if it exists, is unique.

Proof. Burmeister and Dobell (1970, Ch. 9) showed that there exists a nonempty set $Z \subset(0, \infty)$ such that for any $\rho \in Z$ there exists unique $(P, k)$ satisfying

$$
\begin{gathered}
P=F_{x}(0, k), \quad F(\dot{k}, k) \equiv F(x, k), \\
\rho P=F_{k}(0, k) .
\end{gathered}
$$

Hence, there exists unique $(p, k)$ satisfying

$$
\begin{aligned}
p=T_{x}(0, k) & =u^{\prime}[F(0, k)]\left[F_{x}(0, k)\right] \\
\rho p=T_{k}(0, k) & =u^{\prime}[F(0, k)] F_{k}(0, k) \\
( & =\partial H / \partial k) .
\end{aligned}
$$

For the case $\rho=0$, it is easily seen that a steady state exists and is unique under our assumption that the problem $\max _{\{k\}} F(0, k)$ has a unique solution $k^{*}$ with $F\left(0, k^{*}\right)>0$.

Thus, for the no-joint production case the locus of steady states in the $c-\rho$ plane is of the general shape depicted by Figure I. Using (2.5), (2.18), and (2.22), we can examine the relationship between paradoxical steady states and instability. From (2.5)

$$
\frac{d \bar{c}}{d \rho}=F_{k}(0, k) k^{\prime}(\rho)
$$


where $k_{j}^{\prime}(\rho), j=1, \ldots, n$, is finite for the no-joint production case provided that the cost functions have continuous second partial derivatives for feasible $\rho \in Z$.

Now, from (2.18), whenever $M^{-1}$ exists,

$$
\left(\begin{array}{l}
p^{\prime} \\
k^{\prime}
\end{array}\right)=(\operatorname{adj} M)\left(\begin{array}{c}
0 \\
-p
\end{array}\right) / \operatorname{det} M
$$

Thus, $k_{j}^{\prime}(\rho)$ is of the form

$$
k_{j}^{\prime}(\rho)=Z_{i}(\rho) / \operatorname{det} M
$$

and

$$
\begin{aligned}
\bar{c}^{\prime}(\rho) & =\sum_{j} F_{k_{j}}(0, k) Z_{j}(\rho) / \operatorname{det} M \\
& =N(p, k, \rho) / D(p, k, \rho),
\end{aligned}
$$

where

$$
D(p, k, \rho) \equiv \operatorname{det} M(p, k, \rho) .
$$

Consider the following assumption.

ASSUMPTION N. Let $\left(\mathrm{p}^{0}, \mathrm{k}^{0}\right)$ be a steady state at the rate of interest $\rho^{0}+\mathrm{g}$. Then $\overline{\mathrm{c}}^{\prime}\left(\rho^{0}\right)=0$ implies $\mathrm{N}\left(\mathrm{p}^{0}, \mathrm{k}^{0}, \rho^{0}\right)=0$ and $\mathrm{D}\left(\mathrm{p}^{0}, \mathrm{k}^{0}, \rho^{0}\right) \neq 0$, where $\mathrm{N}$ and $\mathrm{D}$ are defined by (3.4) and (3.5). Similarly $\overline{\mathrm{c}}^{\prime}\left(\rho^{0}\right)= \pm \infty$ implies that $\mathrm{D}\left(\mathrm{p}^{0}, \mathrm{k}^{0}, \rho^{0}\right)=0$ and $\mathrm{N}\left(\mathrm{p}^{0}\right.$, $\left.\mathrm{k}^{0}, \rho^{0}\right) \neq 0$. If $\mathrm{D}\left(\mathrm{p}^{0}, \mathrm{k}^{0}, \rho^{0}\right)=0$, then $\mathrm{N}\left(\mathrm{p}^{0}, \mathrm{k}^{0}, \rho^{0}\right) \neq 0$.

Assumption $N$ serves to rule out zero divided by zero indeterminacies, which require the application of l'Hospital's rule, thereby complicating the analysis without adding any economic insights.

The following proposition can now be proved.

Proposition 1. For the no-joint production case, the matrix $\mathrm{J}$ of system (2.15a) has no zero root.

Proof. Since $\operatorname{det} J=\Pi_{i=1}^{2 n} \lambda_{i}$, we need only to show that $\operatorname{det} J=$ $-\operatorname{det} M \neq 0$. It can be shown that $\bar{c}^{\prime}(\rho)$ is finite for the no-joint production case, and this implies that $\operatorname{det} M \neq 0$.

Q.E.D.

While multiple rest points are ruled out, paradoxical steady states may exist, as illustrated in Figure I. Under the following assumptions, we can show that every rest point is a regular saddlepoint.

AsSUMPTION A.1. The steady state $\left(\mathrm{p}^{*}, \mathrm{k}^{*}\right)$ corresponding to $\rho=0$ is a regular saddlepoint. 
In every economic example we have seen the Golden Rule point at $\rho=0$ is a regular saddlepoint, and thus the class of economic models for which our assumption is valid is obviously wide and of significant interest.

AssumPtion A.2. For all $\rho \in \mathrm{Z}$ the matrix $\mathrm{J}(\rho)$ has no pure imaginary roots.

Without Assumption A.2, the inference about the stability properties of a nonlinear system using its associated linear system is not valid. ${ }^{15}$

THEOREM 3. Under A.1 and A.2 all rest points are regular saddlepoints.

Proof. Propositions 1 and A.2 imply that real parts of roots of $J$ do not change sign. (Note that roots of $J$ vary continuously with $\rho$.) Hence the stability properties of all rest points are the same as those of the rest point $\left(p^{*}, k^{*}\right)$ at $\rho=0$. Assumption A.1 completes the proof.

Q.E.D.

Under these conditions the existence of a limit cycle around an unstable saddlepoint (or completely unstable point) is impossible.

\section{The Joint Production CASE With $n>1$}

For the joint production case, for any given $\rho>0$, there may exist more than one steady state. Consider the equations,

$$
\begin{gathered}
\dot{k}=\partial H / \partial p=0 \\
\dot{p}=\rho p-\partial H / \partial k=0,
\end{gathered}
$$

or, more simply,

$$
\psi(\rho, p, k)=0 .
$$

Let $S$ be the solution set of the equations (4.3), that is, $S=\{(\rho, p, k) \mid \rho$ $\left.\geqq 0,(p, k) \in R_{+}^{2 n}, \psi(\rho, p, k)=0\right\}$. We assume that $S$ is nonempty and $S \subset[0, \infty) x\left(R_{++}\right)^{2 n}$, where $R_{++} \equiv(0, \infty)$. Brock (1973) showed that if $\operatorname{det} M \neq 0$ for all $(\rho, p, k) \in S$ and if $(p, k)$ is unique for $\rho=0$, then for given $\rho>0,(p, k)$ is uniquely determined. As we have shown, det

15. See Berger and Berger (1968), pp. 147-48, for an interesting example. In such instances a more complex analysis is required using approximation involving higher order terms of a Taylor series expansion around equilibrium; similar results then can be derived. We also note that if $J$ has a pure imaginary root, the associated linear system will have a closed path about the equilibrium point. We are not aware of any continuous-time economic model with our criterion function for which the optimizing trajectory is a closed path. 
$M \neq 0$ for the no-joint production case. For the joint production case, the assumption $\operatorname{det} M \neq 0$ is too strong; we make only the following assumption.

AsSUMPTION I. $\operatorname{det} \mathrm{M}=0$ only at isolated points in $\mathrm{S}$.

Assumption I ensures that for any $\rho>0$ the set $S_{\rho}=\{(p, k) \mid(\rho$, $p, k) \in S\}$ is discrete.

We shall need the following assumptions:

ASSUMPTION U (local uniqueness of optimal path for a given initial vector $\left.\mathrm{k}_{0}\right)$. For $\rho \geqq 0$, any rest point $(\mathrm{p}, \mathrm{k})$ has at most $\mathrm{n}$ roots with negative real parts.

Assumption $U$ can be justified on the ground that nonuniqueness can be eliminated by dropping the goods that cause it (e.g., blue and green machines).

ASSUMPTION C. For a given (feasible) value $\rho=\rho^{0}>0$, there exist three steady-state consumption levels $\overline{\mathrm{c}}^{1}>\overline{\mathrm{c}}^{2}>\overline{\mathrm{c}}^{3}$ corresponding to the three rest points $\left(\mathrm{p}^{1}, \mathrm{k}^{1}\right),\left(\mathrm{p}^{2}, \mathrm{k}^{2}\right)$, and $\left(\mathrm{p}^{3}, \mathrm{k}^{3}\right)$, as illustrated in Figure II.

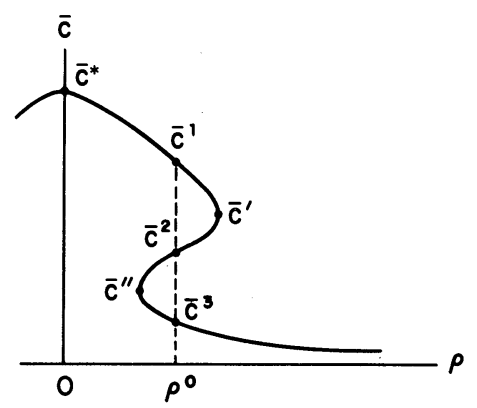

FIGURE II

THEOREM 4. Under assumptions $N, I, U$, and $C$, and excluding inflection points of the $\mathrm{c}-\rho$ graph, if $\left(\mathrm{p}^{1}, \mathrm{k}^{1}\right)$ is a regular saddlepoint, then $\left(\mathrm{p}^{2}, \mathrm{k}^{2}\right)$ is unstable (with less than $\mathrm{n}$ roots having negative real parts).

The proof of Theorem 4 is available from the authors upon request. We cannot say much about $\left(p^{3}, k^{3}\right)$ given only that $\left(p^{1}, k^{1}\right)$ is stable. However, if we know that $\left(p^{3}, k^{3}\right)$ is a regular saddlepoint as well as $\left(p^{1}, k^{1}\right)$, then we can state the following. 
THEOREM 5 (separation theorem). The points of stable equilibrium (in the saddlepoint sense) $\left(\mathrm{p}^{1}, \mathrm{k}^{1}\right)$ and $\left(\mathrm{p}^{3}, \mathrm{k}^{3}\right)$ are separated by a point of unstable equilibrium $\left(\mathrm{p}^{2}, \mathrm{k}^{2}\right)$.

It is interesting to note that if $n$ is even, then $\left(p^{2}, k^{2}\right)$ is not completely unstable. This result seems quite reasonable. For the case $n=2$, apparently the policy functions $\dot{k}_{i}=\dot{k}_{i}\left(k_{1}, k_{2}\right)$ are as depicted in Figure III, where in general the $n$ policy functions $\dot{k}=\dot{k}(k)$ represent the solution to the synthesis problem, i.e., the optimal control law in feedback form.

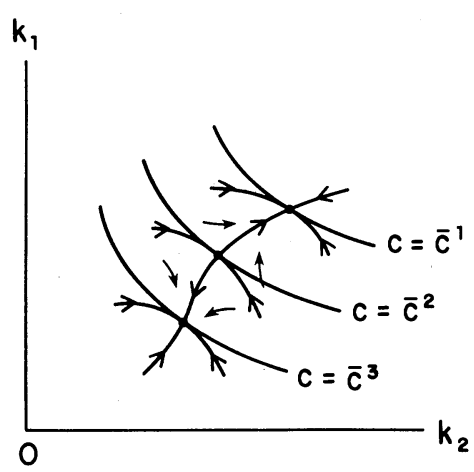

Figure III

Finally, suppose that assumptions (A.1), (A.2), U, and N are satisfied, and suppose also that no two characteristic roots of $J$ vanish simultaneously; then the following proposition holds (proof available from the authors).

PROPOSITION. If for all $\rho \geqq 0$ there are no multiple rest points corresponding to a given $\rho$, then all rest points for which det $\mathrm{J}$ $\neq 0$ are regular saddlepoints in the sense of Definition 3.

This result represents a Correspondence Principle because multiple rest points cannot occur for economies that are regular in the sense of Definition 2. Moreover, an extension to this proposition may be proved that represents a verification of Conjecture $\mathrm{CP}$ under the stated assumptions.

Consider the locus of steady-state points $(c, \rho)$ in Figure II. Those points $(c, \rho)$ on this locus for which $c>\bar{c}^{\prime}$ (and hence not paradoxical in the sense of Definition 1) correspond to regular saddlepoints in $(p, k)$ space. Like- 
wise those points $(c, \rho)$ such that $\bar{c}^{\prime \prime}<c<\bar{c}^{\prime}$ (and thus paradoxical) are unstable in the sense of Definition 3.

\section{THE SOCIAL RATE OF RETURN}

Solow $(1963,1967)$ has introduced the concept of the social rate of return to savings-investment and has proved that under quite general conditions this social rate of return equals the interest rate. Specifically, for convenience we first outline a discrete-time variant of our model with a single consumption good. Consider an economy that has been in a steady-state equilibrium up to period $t=0$ with per capita consumption $c(t)=\bar{c}$, per capita capital stock vector $k(t)=$ $\bar{k}$, price vector $\bar{p}$, and interest rate $r(t)=\bar{r}$. Starting from these initial conditions, assume that prices remain at $\bar{p}$, and let

$$
\{c(t)\}_{t=0}^{\infty}
$$

be a feasible consumption stream that is also efficient in the sense that $c\left(t^{\prime}\right)>c(t)$ is impossible unless $c\left(t^{\prime \prime}\right)<c(t)$ for some other time period $t^{\prime \prime}$. Solow (1967, especially pp. 34-35) then has proved that, if the economy approaches a new steady-state equilibrium with $c=c^{*}, k$ $=k^{*}, p=p^{*}=\bar{p}$, and $r=r^{*}=\bar{r}$, then the present discounted value of the increment stream,

$$
z(0)=\frac{z(1)}{1+\theta}+\cdots+\frac{z(t)}{(1+\theta)^{t}}+\cdots=0, z(t)=c(t)-\bar{c}
$$

has a $\operatorname{root} \theta=\bar{r}$. Analogously it can be shown that in continuous time

$$
\int_{0}^{\infty} z(t) e^{-\theta t} d t=0
$$

has a $\operatorname{root} \theta=\bar{r}$. (In this section we set $g=\dot{L} / L=0$ for convenience.) We are interested in nontrivial cases for which $\bar{c} \neq c^{*}$. But $\bar{c}$ and $c^{*}$ both represent steady-state consumption at identical prices $\bar{p}=p^{*}$; in this case there must exist a linearity in the production of the consumption good, thereby violating strict concavity of the technology or the assumption that the consumption good is produced by a neoclassical production function. For this reason we now consider transitions with $\bar{p} \neq p^{*}$.

An economy has a rate of time preference $\rho>0$ and a social welfare function,

$$
W=\int_{0}^{\infty} c(t) e^{-\rho t} d t
$$


Suppose that this economy is initially in a steady-state equilibrium with $c=\bar{c}, k=\bar{k}, p=\bar{p}$, and $r=\bar{r}$. We now no longer take prices as fixed at $\bar{p}$, but rather suppose that there exists a feasible and efficient consumption stream,

$$
\{\hat{c}(t)\}_{t=0}^{T}
$$

such that the transition terminates at time $t=T$ in a new steady-state equilibrium with

$$
c(T)=c^{*}, k(T)=k^{*}, p(T)=p^{*} \text {, and } r(T)=r^{*} .
$$

Further, suppose that this new steady state is "adjacent" to the initial one with

$$
\left|c^{*}-\bar{c}\right|<\epsilon
$$

and

$$
\left|r^{*}-\bar{r}\right|<\epsilon,
$$

where $\epsilon$ is an arbitrarily small positive number. Finally, if there exists a feasible and efficient finite-time transition from the steady state $(\bar{r}, \bar{c})$ to $\left(r^{*}, c^{*}\right)$, then we assume that there exists a reverse transition from $\left(r^{*}, c^{*}\right)$ to $(\bar{r}, \bar{c})$,

$$
\left\{c^{0}(t)\right\}_{t=0}^{\infty}
$$

such that

$$
c^{0}(t)-c^{*}=-[\hat{c}(t)-\bar{c}], \quad t \geqq 0 .
$$

Actually, an alternative and weaker condition will suffice for our purposes, namely, the condition that every efficient transition (5.5) from a steady state with consumption $\bar{c}$ to one with consumption $c^{*}$ has the property that the transition would be preferred, on welfare grounds, to remaining in the initial steady state if the rate of time preference were in fact $r^{*}$, i.e., $\hat{c}(t)$ must satisfy

$$
\int_{0}^{\infty} \hat{c}(t) e^{-r^{*} t} d t>\int_{0}^{\infty} \bar{c} e^{-r^{*} t} d t
$$

While the condition (5.9b) seems more obscure than (5.9a), they are interchangeable in all that follows.

Note that we are assuming here that efficient transitions between these two "adjacent" steady states are possible, and that there exist such transitions satisfying (5.5)-(5.9a) or, alternatively, (5.5)-(5.7) and (5.9b). The technical problems involved to ensure that these conditions will be met are far from trivial, but they are not of economic 
consequence for our expositional purposes in this section. Moreover, it is clear that transitions meeting all of our requirements do exist for a Leontief-Sraffa technology with alternative techniques of production (see Solow (1967)). With this important warning we proceed with our story.

Should our imaginary economy undertake the transition just described? The answer to this question depends in part on the relationship between $\bar{c}, \bar{r}, c^{*}, r^{*}$, and $\rho$. As a first step toward finding an answer, we prove a slight generalization of Solow's Theorem.

THEOREM 6. Consider finite-time transitions satisfying (5.5)-(5.9a), or (5.5)-(5.7) and (5.9b), and define

$$
\mathrm{f}(\theta) \equiv \int_{0}^{\infty}[\hat{\mathrm{c}}(\mathrm{t})-\overline{\mathrm{c}}] \mathrm{e}^{-\theta \mathrm{t}} \mathrm{dt}
$$

Assume that the technology $\mathrm{c},=\mathrm{F}(\dot{\mathrm{k}}, \mathrm{k})$ is strictly concave in $\dot{\mathrm{k}}$. Then the equation $\mathrm{f}(\theta)=0$ has a root $\theta=\mathrm{r}$ such that $\mathrm{r} \in(\overline{\mathrm{r}}$, $\left.\mathrm{r}^{*}\right)$. We shall refer to such an $\mathrm{r}$ as a social rate of return for the associated transition.

Proof. It is well-known that the steady-state point $(\bar{r}, \bar{c})$ would solve problem (1) given $k(0)=\bar{k}, u(c)=c$, and $\rho=\bar{r}$. Likewise $\left(r^{*}, c^{*}\right)$ is an optimal rest point when $k(0)=k^{*}, u(c)=c$, and $\rho=r^{*}$. Thus, from (5.10) and (5.9a) we see that

$$
f(\bar{r})<0
$$

and

$$
f\left(r^{*}\right)>0,
$$

where the strict inequality follows because $F_{x x}, x=\dot{k}$ is negative definite almost everywhere. Since $f(\theta)$ is continuous, there exists a root $\theta=r$ such that $f(r)=0$ and $r \in\left(\bar{r}, r^{*}\right)$. Alternatively, (5.9b) implies (5.12).

Q.E.D.

Theorem 6 might lead one to misuse of Solow's social rate of return concept if no additional restrictions are placed on the transitions under consideration. For example:

\section{Naive Rule}

A transition from the steady state $(\bar{r}, \bar{c})$ to $\left(r^{*}, c^{*}\right)$, which satisfies (5.5)-(5.7) should be undertaken provided that $\bar{r}$ and $r^{*}$, and hence a social rate of return $r$, exceed the rate of time preference $\rho$ (provided that $\rho$ exceeds $\bar{r}$ and $r^{*}$, and hence $r$ ), and provided that the new steady 


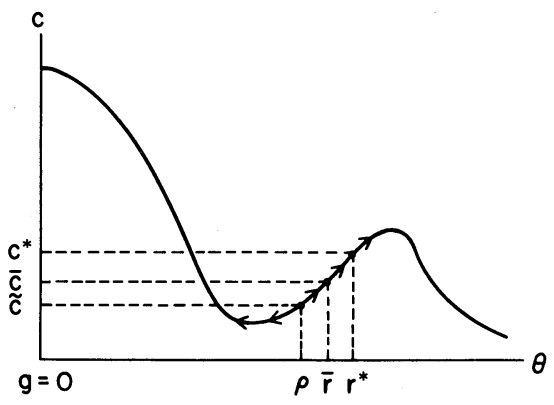

FIGURE IV

state has higher per capita consumption with $c^{*}>\bar{c}$ (and provided that $c^{*}<\bar{c}$ ).

An economy that follows this Naive Rule exhibits an interesting Correspondence Principle similar to our Conjecture CP, namely, it will converge to the correct optimal rest point that solves problem (1) if the economy is regular in the sense of Definition 2. On the other hand, if the optimal steady-state point $(\rho, \tilde{c})$ is paradoxical in the sense of Definition 1, then $(\rho, \tilde{c})$ is unstable in that, starting from an initial steady-state point $(\bar{r}, \bar{c})$ arbitrarily close, an economy following the Naive Rule will move away from $(\rho, \tilde{c})$. These results are illustrated in Figures IV and V.

When is the Naive Rule reasonable? Clearly a transition satisfying the conditions of the Naive Rule is desirable on legitimate welfare grounds if and only if the welfare increments for transitions from $(\bar{r}, \bar{c})$ to $\left(r^{*}, c^{*}\right)$ are positive; these welfare increments are equal

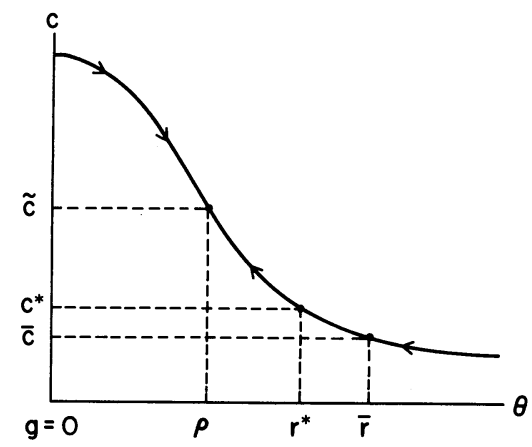

Figure V 
to $f(\rho)$, where $f(\theta)$ is defined by (5.10). Suppose, for example, that the social rate of return $\theta=r$ is unique, as indicated in Figure VI for

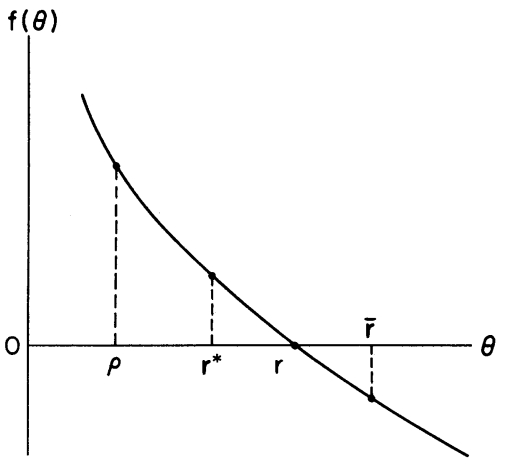

FigURE VI

transitions from $(\bar{r}, \bar{c})$ to $\left(r^{*}, c^{*}\right)$ with $c^{*}>\bar{c}$. In this case we know that $f(0)=\infty$ since $c^{*}>\bar{c}$; but from the proof of Theorem 6 , we also have $f\left(r^{*}\right)>0$ and $f(\bar{r})<0$. Thus, $f(\rho)>0$ when $\rho$ is less than both $r^{*}$ and $\bar{r}$, while $f(\rho)<0$ when $r^{*}$ and $\bar{r}$ both exceed $\rho$.

An intuitively appealing class of transitions does have unique social rates of return. Thus, suppose that the transition (5.5) also satisfies

$$
\left\{\begin{array}{l}
\hat{c}(t) \leqq \bar{c}, \quad t \in[0, \hat{t}] \\
\hat{c}(t) \geqq \bar{c}, \quad t \in[\hat{t}, \infty) \\
\hat{c}(t)=c^{*}>\bar{c}, \quad t \in[T, \infty),
\end{array}\right.
$$

as pictured in Figure VII. When transitions satisfy (5.13), it is straightforward to prove that any root of $f(\theta)=0$ is unique. The crucial

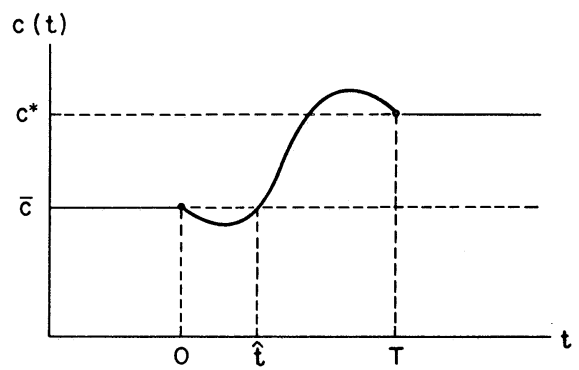

Figure VII 
aspect of such transitions is that there are two phases: The first phase may be identified with a "consumption sacrifice" that allows the economy eventually to attain a higher level of steady-state consumption $c^{*}>\bar{c}$; the second phase is characterized by $c(t) \geqq \bar{c}$. Similarly, any transition from a high steady-state level of consumption to a lower one will have a unique rate of return if it consists of two phases: the first phase is a "consumption binge," which results, in phase two, in a lower consumption level than the original one.

However, even if the social rate of return $r$ is not unique, for regular economies we can establish, when (5.5)-(5.9a) (or (5.5)-(5.7) and $(5.9 \mathrm{~b}))$ hold, that transitions to a steady state sufficiently close to $(\rho, \tilde{c})$ will have an associated welfare increment that is positive. To see this fact, consider an initial steady state $(\bar{r}, \bar{c})$ with $\bar{c}\langle\tilde{c}$ and $\bar{r}\rangle$ $\rho$. Because the economy is regular, there exists a steady state $\left(r^{*}, c^{*}\right)$ with $\rho<r^{*}<\bar{r}$ and $c>c^{*}>\bar{c}$. As argued above, $f\left(r^{*}\right)>0$ for this transition, and thus we may select a $c^{*}$ sufficiently close to $\tilde{c}$ that $f(\rho)$ $>0$. In this case the Naive Rule improves welfare. We emphasize that this result does not depend upon the uniqueness of the social rate of return for transitions.

Unfortunately, with irregular economies these apparently plausible results no longer remain valid. Thus, consider an irregular economy for which the rest points corresponding to all $\theta \in\left[\rho, r^{*}\right]$ are paradoxical in the sense of Definition 1 . Now a transition satisfying (5.5)-(5.9a) (or (5.5)-(5.7) and (5.9b)) with $c^{*}>\bar{c}$ must entail $r^{*}>$ $\bar{r}$ because a rise in steady-state consumption is accompanied by a rise in the steady-state interest rate when there is paradoxical behavior. Therefore, we see that $f(\theta)$ defined by (5.10) must be as illustrated in Figure VIII with more than one root to $f(\theta)=0$; this fact follows immediately from the continuity of $f(\theta), f(0)>0, f(\bar{r})<0, f\left(r^{*}\right)>0$, and

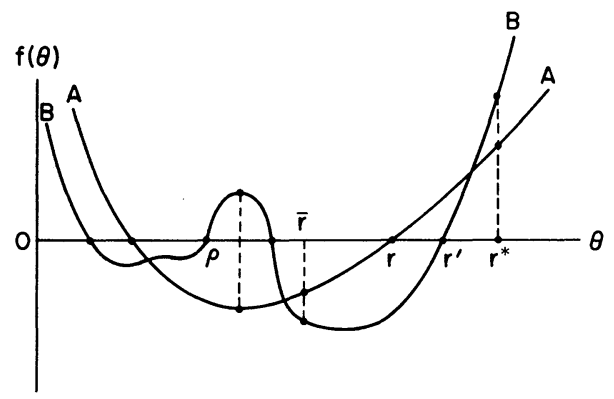


$r^{*}>\bar{r}$. Accordingly, under these circumstances transitions between adjacent paradoxical steady states that have a unique social rate of return do not exist. Hence for a feasible transition from $\bar{c}$ to $c^{*}>$ $\bar{c}$, satisfying (5.5)-(5.9a) (or (5.5)-(5.7) and (5.9b)), we have $c(t)=\bar{c}$ at more than one value of $\hat{t}>0$. (In particular, such transitions also satisfying (5.13) do not exist between adjacent paradoxical steady states). Conversely, if there exist transitions satisfying (5.5)-(5.9a) (or (5.5)-(5.7) and (5.9b)), which have a unique social rate of return, the associated steady states cannot be paradoxical.

\section{CONCLUDING REMARKS}

For practical economic policy, one cannot expect to solve optimal control problems of the form (1). On the other hand, it is quite reasonable to suppose that an actual economic policy will be implemented if the calculated benefits exceed estimated costs. We have seen that such cost-benefit planning is potentially quite dangerous if it is based upon rate-of-return calculations and the social rate of return is not unique. In this case the economy may move away from the true rest point solution for the appropriate criterion, i.e., for problem (1), if the economy is not regular. However, the Burmeister-Turnovsky (1972) concept of a regular economy does provide some rationale for the Naive Rule based on rates of return; if the economy is regular (i) such a Naive Rule results in convergence to the appropriate, i.e., truly optimal, steady state, and (ii) there exists a $c^{*}$ sufficiently close to the optimal consumption level $\tilde{c}$ such that a transition from $\bar{c}$ to $c^{*}$ entails a strictly positive welfare increment.

For descriptive economics the Hahn instability problem still exists, but it can be argued that the Hahn formulation in which actual and expected rates of price change always coincide is an incorrectly specified model. Once price expectations are introduced, a descriptive heterogeneous capital good model may be stable; see Burmeister and Graham (1974). But this is quite another story.

The existence of many capital goods $i s$ a severe complication. A powerful Correspondence Principle, our Conjecture CP, is valid when $n=1$; see Theorems 1 and 2. But it does not follow that neoclassical parables lose all relevance when heterogeneous capital goods exist, i.e., when $n>1$. Rather, we now require additional assumptions to establish the same qualitative conclusions; see Theorems 3 and 5 . When the economy is regular, thereby excluding paradoxical behavior, and when every rest point to problem (1) is a regular saddlepoint, 
much of the conventional wisdom based on the one-capital good case again prevails. We find this reassuring.

\author{
UNIVERSITY OF VIRGINIA \\ AUSTRALIAN NATIONAL UNIVERSITY
}

\title{
REFERENCES
}

Berger, Melvyn, and Marion Berger, Perspectives in Nonlinearity (New York: W. A. Benjamin, Inc., 1968).

Blaug, Mark, The Cambridge Revolution: Success or Failure? (London: The Institute of Economic Affairs, 1975).

Brock, William A., "Some Results on the Uniqueness of Steady States in Multisector Models of Optimum Growth When Future Utilities Are Discounted," International Economic Review, XIV (Oct. 1973), 535-59.

_- , and Edwin Burmeister, "Regular Economies and Conditions for Uniqueness of Steady States in Optional Multi-Sector Economic Models," International Economic Review, XVII (Feb. 1976), 105-20.

Brown, M., K. Sato, and P. Zarembka, eds., Essays in Modern Capital Theory (Amsterdam: North-Holland Publishing Company, 1976).

Burmeister, Edwin, and A. Rodney Dobell, Mathematical Theories of Economic Growth (New York: The Macmillan Company, 1970).

, and D. A. Graham, "Multi-Sector Economic Models with Continuous Adaptive Expectations," Review of Economic Studies, XLI (July 1974), 323-36.

_- , and P. J. Hammond, "Maximin Paths of Heterogeneous Capital Accumulation and the Instability of Paradoxical Steady States," Econometrica, forthcoming 1977.

__, and S. J. Turnovsky, "Capital Deepening Response in an Economy with Heterogeneous Capital Goods," American Economic Review, LXII (Dec. 1972), 84253.

Cass, David, and Karl Shell, "The Structure and Stability of Competitive Dynamical Systems," Journal of Economic Theory, XII (Feb. 1976), 31-70.

Hahn, F. H. "Equilibrium Dynamics with Heterogeneous Capital Goods," this Journal, LXXX (Nov. 1966), 633-46.

Harcourt, G. C., Some Cambridge Controversies in the Theory of Capital (London: Cambridge University Press, 1972).

Kurz, M., "The General Instability of a Class of Competitive Growth Processes," Review of Economic Studies, XXXV (April 1968), 155-74.

Levhari, David, "A Nonsubstitution Theorem and Switching of Techniques," this Journal, LXXIX (Feb. 1965), 98-105.

Liviatan, Nissan, and Paul A. Samuelson, "Notes on Turnpikes: Stable and Unstable," Journal of Economic Theory, III (Dec. 1969), 454-75. Reprinted in Robert C. Merton, ed., The Collected Scientific Papers of Paul A. Samuelson, Volume III (Cambridge, Mass.: The MIT Press, 1972).

"Paradoxes in Capital Theory: A Symposium," this Journal, LXXX (Nov. 1966), 503-83.

Robinson, Joan, The Accumulation of Capital (New York: St. Martin's Press, 1966).

Samuelson, Paul A., "The General Saddlepoint Property of Optimal Control Motions," Journal of Economic Theory, V (Aug. 1972), 102-20.

_- , "Indeterminacy of Development in a Heterogeneous-Capital Model with Constant Saving Propensity," in K. Shell, ed., Essays on the Theory of Optimal Economic Growth (Cambridge, Mass.: MIT Press, 1967), 219-31.

_- "The Stability of Equilibrium: Comparative Statics and Dynamics," Econometrica, IX (April, 1941), 97-120. Reprinted in Joseph E. Stiglitz, ed., The Collected Scientific Papers of Paul A. Samuelson, Volume I.(Cambridge, Mass.: MIT Press, 1966).

_- "The Stability of Equilibrium: Linear and Non-Linear Systems," Econometrica, X (Jan. 1942), 1-25. Reprinted in Joseph E. Stiglitz, ed., The Collected Scientific Papers of Paul A. Samuelson, Volume I (Cambridge, Mass.: MIT Press, 1966). 
Shell, Karl, and J. E. Stiglitz, "The Allocation of Investment in a Dynamic Economy," this Journal, LXXXI (Nov. 1967), 592-609.

Solow, Robert M., Capital Theory and the Rate of Return (Amsterdam: North-Holland Publishing Company, 1963).

-_, "The Interest Rate and Transition Between Techniques," in C. H. Feinstein, ed., Socialism, Capitalism and Economic Growth (New York: Cambridge University Press, 1967), 30-39. 